\title{
HERRAMIENTAS TECNOLÓGICAS APLICADAS AL CONOCIMIENTO Y APLICACIÓN DEL DERECHO PENAL
}

\section{TECHNOLOGICAL TOOLS APPLIED TO THE KNOWLEDGE AND APPLICATION OF CRIMINAL LAW}

\author{
José Manuel Palma Herrera ${ }^{1}$ \\ Fecha de recepción: 11/07/2019; Fecha de revisión: 15/07/2019; Fecha de aceptación: 25/07/2019 \\ Cómo citar este artículo: \\ Palma Herrera, J.M. (2019). Herramientas tecnológicas aplicadas al conocimiento y aplicación del Derecho \\ Penal. Revista de Innovación y Buenas Prácticas Docentes, 8(3), 20-27. \\ Autor de Correspondencia: jmpalma@uco.es
}

\begin{abstract}
Resumen:
Se trata de una experiencia con la que se pretende formar al alumno en el uso de las nuevas herramientas tecnológicas que permiten el acceso a las interpretaciones aplicativas que la jurisprudencia viene haciendo de los conceptos básicos de la disciplina penal, conceptos previamente seleccionados por el profesorado como parte del proyecto docente. Mediante el trabajo en grupos de tamaño reducido, los alumnos construyen el contenido de tales conceptos a partir del análisis y discriminación de las posiciones jurisprudenciales que encuentran utilizando diversos recursos electrónicos, consiguiendo, de esta forma, mejorar su proceso de aprendizaje en dos aspectos sustanciales: 1) facilitándoles la comprensión de los conceptos esenciales de la disciplina y de los problemas aplicativos que plantean y 2) ayudándoles a superar la fractura existente entre la teoría que se imparte a lo largo de la carrera y la realidad a la que tiene que hacer frente el profesional. Los resultados positivos alcanzados hacen de éste un método de trabajo altamente recomendable en disciplinas que forman parte de lo que se denomina "Derecho positivo" que, por estar sujetas a continuos cambios normativos, requieren del profesional del Derecho un proceso de constante actualización y puesta al día en sus conocimientos.
\end{abstract}

Palabras clave: autoaprendizaje, bases de datos, Derecho penal, jurisprudencia.

\begin{abstract}
:
This project is an experience that aims to train students in the use of new technological tools, which allow them to know the jurisprudential interpretation of the basic concepts of criminal law. The students, working in small groups and using electronic resources, build those concepts by detecting and analyzing the jurisprudential positions. In this way, students manage to improve their learning process in two key aspects: 1) getting a better understanding of the key concepts of the field and their application problems, and 2) being helped to overcome the existing fracture between the theoretical knowledge and the practical application of the law. This method of working is highly recommended for the Law, given the positive results obtained, particularly for those legal disciplines, which are constantly changing and that require from professionals a lifelong updating.
\end{abstract}

Key Words: self-instruction, database, criminal law, jurisprudential interpretation

I Universidad de Córdoba (España), jmpalma@uco.es; CÓDIGO ORCID: 0000-0003-0945-4052 


\section{INTRODUCCIÓN}

Los planes de estudio correspondientes al Grado en Derecho han supuesto una disminución significativa del número de créditos dedicados al estudio de la teoría jurídica del delito y de las singulares figuras delictivas en el marco de las asignaturas Derecho Penal I, Derecho Penal II, Derecho Penal III y Derecho Penal Económico con los problemas formativos que ello conlleva (Barquín-Sánchez, 2016).

Frente a esta disminución, los conceptos fundamentales que constituyen el núcleo de esa materia, lejos de contraerse, cada vez se tornan más complejos y cambiantes, sobre todo en el marco de los continuos procesos de revisión del Código Penal a los que venimos asistiendo legislatura tras legislatura. Todo ello genera en el profesional del Derecho la necesidad, cada vez más acuciante, de insertarse en una dinámica de constante revisión y actualización de sus conocimientos.

A lo anterior, hemos de añadir el papel tan extraordinario que en la aplicación práctica del Derecho Penal ha adquirido, por un lado, el tema de la sucesión temporal de leyes, y por otro, la jurisprudencia.

Respecto a lo primero, la vorágine normativa en la que nos vemos inmersos y la lentitud de la justicia penal obliga frecuentemente a tener que valorar de forma comparativa dos y hasta tres regulaciones distintas sobre una misma cuestión (con sus correspondientes interpretaciones y criterios de aplicación) a fin de comprobar cuál de ellas sirve de manera más favorable a los intereses que se defienden. Por lo que respecta a la jurisprudencia, podríamos afirmar, sin riesgo a equivocarnos, que el estudio de la doctrina penal ha quedado relegado, en buena medida, al ámbito de la investigación jurídica, mientras que en el terreno aplicativo del Derecho Penal la principal fuente de estudio para el profesional son las interpretaciones que vienen haciendo nuestros jueces y tribunales a la hora de aplicar los distintos preceptos del Código Penal, convirtiendo así a la jurisprudencia en fuente de conocimiento de primer orden para el jurista (Santaella-Quintero, 2016).

Aunque no dejaremos de insistir en la necesidad de estudiar a la doctrina, pues sólo un conocimiento profundo de los conceptos fundamentales permite al profesional del Derecho construir mentalmente el "edificio" de la teoría jurídica del delito que le habilitará para encauzar con garantías cualquier problema de índole penal al que se enfrente en su vida laboral, sería absurdo no querer ver la realidad de las cosas. Y esa realidad pasa por reconocer que, en ese proceso de permanente formación, de autoaprendizaje, en el que deberá mantenerse el profesional del Derecho a lo largo de toda su vida y que constituye uno de los pilares básicos sobre los que se construye el proceso de Bolonia, juega un papel fundamental el recurso a las fuentes legales y jurisprudenciales que están llenando de contenido buena parte de los conceptos de la disciplina. 
Hoy por hoy, existen a disposición, tanto del alumnado como de los profesionales del Derecho, numerosas bases de datos electrónicas de legislación, jurisprudencia, formularios, etc., que constituyen una extraordinaria herramienta formativa cuyo uso debería ser constante tanto por unos como por otros. Sin embargo, a lo largo de diferentes cursos he podido constatar, al menos por lo que respecta a los alumnos, que esto no era así; que el uso que se hacía de las mismas era muy deficiente como pudo comprobarse en su día gracias a la ejecución del Proyecto de Innovación Docente titulado "APRENDIZAJE DEL DERECHO PENAL A TRAVÉS DE CASOS PROPORCIONADOS POR LA JURISPRUDENCIA" y coordinado por quien suscribe. EI desarrollo de este Proyecto puso de manifiesto que numerosos alumnos tenían serias dificultades para el acceso a esas bases de datos por no conocer de su existencia, no saber utilizarlas a la hora de realizar búsquedas o, realizadas esas búsquedas, no saber extraer de las mismas la información útil para la consecución del fin pretendido. Unas carencias, en definitiva, que sin duda condicionan el uso de este tipo de recursos una vez terminen sus estudios de Derecho, pues si no se les instruye en estas competencias durante su formación universitaria, difícilmente las alcanzarán una vez se inserten en el mundo laboral (Ferrer-Martín de Vidales, 2016).

Los excelentes resultados alcanzados con aquel Proyecto constituyeron un aliciente para seguir insistiendo en esa línea docente dirigida al autoaprendizaje, pero prestando aún más atención, si cabe, al uso de las herramientas tecnológicas a las que antes aludía, que constituyen un instrumento esencial para esa labor de reciclaje y formación continua en los conceptos penales, y que justifican que ya durante el curso 2016/17 se presentase un nuevo Proyecto de Innovación Docente en el que se afrontaba, decididamente, la formación del alumnado en el uso de las nuevas tecnologías aplicadas al conocimiento del Derecho Penal, línea en la que se ha pretende insistir con un nuevo Proyecto a lo largo del curso 2017/18.

Para la adquisición de esa capacidad de autoaprendizaje, que constituye una pieza fundamental en la formación continua y permanente de todo jurista (tal y como pusieron de manifiesto las Conclusiones de la Asamblea de Decanos/as de las Facultades de Derecho españolas, reunida en Córdoba los días 25 y 26 de Octubre de 2007), y que forma parte las competencias genéricas y específicas del Título de Graduado en Derecho, se torna extremadamente útil el sistema de aprendizaje basado en casos reales extraídos de la jurisprudencia, pues forma al alumno en las habilidades y competencias que deberá ejercitar una vez inserto en el terreno laboral. Es, en definitiva, una manifestación del método de aprendizaje basado en problemas (ABP), que permite combinar la adquisición de conocimientos con el aprendizaje de competencias, y en el que los alumnos se ven obligados a investigar y reflexionar para buscar una solución ante un problema planteado por el profesor (López-Guede, 2015; Vizcarro \& Juárez, 2008). 
Sobre la base de estos presupuestos, el Proyecto de innovación docente que se presentó, que se extendería a dos de las cuatro asignaturas que impartieron a lo largo del curso 2017/18 los participantes en el Proyecto (Derecho Penal I y Derecho Penal II), lo que pretendía era formar al alumnado (aproximadamente unos 150) en el uso de las herramientas tecnológicas que resultasen útiles en el proceso de autoaprendizaje basado en problemas aprovechando, al mismo tiempo, las ventajas que ofrece este sistema de formación para que el alumno, a través del estudio y análisis de casos reales extraídos de la jurisprudencia, llegase a adquirir las competencias necesarias para construir los conceptos básicos del Derecho Penal y para reciclar sus conocimientos conforme los cambios legislativos y jurisprudenciales lo fuesen exigiendo. Se proponía implantar, por tanto, una metodología docente en la que se invirtiesen los términos del proceso formativo del alumno, que si tradicionalmente había venido adquiriendo en primer lugar unos conocimientos teóricos facilitados por el profesor, que luego trataba de aplicar al caso concreto, ahora comienza por estudiar un caso concreto para extraer de la solución jurisprudencial que se hubiere dado al mismo aquellos conceptos teóricos básicos que serían reforzados y enriquecidos ya en un segundo estadio por el profesor, que pasa así a desempeñar una labor de depuración, aclaración y enriquecimiento teórico de tales conceptos con las aportaciones que la doctrina, siempre necesaria, haya venido haciendo.

La elección de las asignaturas Derecho Penal I y Derecho Penal II obedece al hecho de ser asignaturas en las que se estudian los conceptos básicos de la disciplina sobre los que la jurisprudencia se ha pronunciado de forma prolija, y respecto a las que ha venido haciendo interesantísimas interpretaciones que son de aplicación diaria en nuestros juzgados y tribunales, conceptos e interpretaciones que los estudiantes van a tener que utilizar el día que, ya como profesionales, se incorporen a la práctica forense.

Se trata de asignaturas en las que los participantes en el Proyecto han venido utilizando la plataforma Moodle desde su implantación en la Universidad de Córdoba, disponiendo a tal efecto de unos materiales ya elaborados y de una experiencia que pone de manifiesto su interés por la calidad en la docencia (así lo evidencian las excelentes calificaciones obtenidas en el Programa Docentia) y que resultan fundamentales para la consecución de los objetivos que se proponen.

Los objetivos que se perseguían eran los siguientes:

1. Formar al alumno en el uso de las nuevas herramientas tecnológicas que permiten el acceso a las reformas de la legislación penal y a las interpretaciones aplicativas que la jurisprudencia viene haciendo de los conceptos básicos de la disciplina penal.

2. Mejorar el proceso de aprendizaje del alumnado facilitando la comprensión de esos conceptos esenciales y de los problemas aplicativos que plantean, comprensión que sin duda resulta mucho más fácil sobre la base de casos reales.

3. A través de la mejor comprensión de esos conceptos básicos, mejorar los resultados académicos del alumnado, pero también asentar tales conceptos sobre pilares más sólidos.

4. Formar al alumno en las competencias de autoaprendizaje a través de las técnicas de análisis e interpretación de las resoluciones jurisprudenciales, técnicas que le dotarán de la capacidad necesaria para mantener actualizados sus conocimientos a lo largo de toda su carrera profesional. 
6. Construyendo el conocimiento teórico desde la práctica, se pretende contribuir, asimismo, a acabar con uno de los motivos de mayor crítica a los estudios de Derecho: la fractura existente entre la teoría que se imparte a lo largo de la carrera, y la realidad a la que tiene que hacer frente el profesional.

\section{DESARROLLO DE LA EXPERIENCIA DE INNOVACIÓN}

\subsection{Material empleado}

Los materiales empleados han sido los previstos inicialmente. Básicamente las bases de datos de legislación y jurisprudencia a las que está suscrita la universidad de Córdoba (básicamente, Aranzadi y Tirant Online), así como alguna otra de naturaleza gratuita que han utilizado también los alumnos, como la del CENDOJ (Centro de Documentación Judicial dependiente del Consejo General del Poder Judicial). A las anteriores, hay que añadir las herramientas de naturaleza ofimática Word y Power Point, así como Adobe Acrobat.

Se ha utilizado, asimismo, el equipamiento informático disponible en el Aula 20 de la Facultad de Derecho y Ciencias Económicas y Empresariales, que era el espacio físico donde se ha desarrollado la experiencia, así como la plataforma Moodle, a la que los alumnos han debido subir todo el material de trabajo elaborado.

\subsection{Método seguido}

En cuanto al método empleado, se ha ajustado fielmente a lo previsto en el proyecto presentado en su día:

1) En primer lugar, hice una selección de conceptos básicos sobre los que los alumnos debían trabajar. La selección se hizo teniendo en cuenta, además, la disponibilidad temporal y los plazos en las que se debía ir desarrollando la experiencia.

2) Se seleccionaron un total de 27 conceptos:

Tentativa inidónea,

Desistimiento

Dominio del hecho

Autoría mediata

Inducción

Inducción en cadena

Cooperación necesaria

Complicidad

Participación del extraneus

Principio de especialidad

Principio de consunción

Concurso real de delitos

Concurso ideal de delitos

Concurso medial de delitos

Delito continuado

Delito masa

Confesión de la infracción a las autoridades

Reparación del daño o disminución de sus efectos

Dilaciones indebidas

Alevosía y abuso de superioridad

Uso de disfraz 
Precio, recompensa o promesa

Abuso de confianza

Circunstancia mixta de parentesco

Fines de la pena

Asesinato con ensañamiento

Asesinato con alevosía

3) Seleccionados esos conceptos básicos, se abrió la posibilidad de participar en la experiencia en grupos reducidos de alumnos, posibilidad que tuvo una extraordinaria acogida por parte del alumnado, participando un total de 82 alumnos.

4) Los trabajos se han realizado en grupos de 3 alumnos. Sólo excepcionalmente se permitieron grupos de alumnos superiores, que en ningún caso superaran los 5.

5) La participación fue voluntaria, computando como actividad de clase en la nota final de la asignatura.

6) La participación suponía respetar escrupulosamente los plazos establecidos para la realización y exposición de los trabajos salvo casos de fuerza mayor, y sin que en ningún caso se considerase fuerza mayor (dada la experiencia de otros años) la realización de exámenes, trabajos, etc., correspondientes a otras asignaturas. De cara a la exposición en clase del trabajo realizado, tampoco se ha considerado causa de fuerza mayor la coincidencia horaria con asignaturas de otros cursos (en el caso de alumnos repetidores).

7) El incumplimiento de lo previsto en el punto 3 suponía la expulsión del grupo de este tipo de actividades, así como su calificación con la nota de 0 . Sin embargo, no se ha producido ningún incumplimiento, resultando así la experiencia mucho más satisfactoria que en cursos anteriores.

8) Cada grupo de trabajo se comprometió a:

a. Buscar al menos tres resoluciones judiciales recientes sobre los contenidos que le hubieren sido asignados.

b. Remitir al profesor responsable de curso, con carácter previo y a través de la plataforma Moodle, tanto las resoluciones encontradas, como el material informático que se fuese a utilizar como base para las exposiciones en clase.

c. Exponer en clase cada semana en la hora establecida en el cronograma elaborado y con apoyo del material informático que considerase necesario (PDF, PowerPoint...) cada una de las resoluciones buscadas, con indicación de los siguientes aspectos relativos a las mismas:

i. Identificación de la resolución:

1. Fecha y número de la resolución.

2. Órgano judicial que la dictó.

3. Referencia de la base de datos de la que se haya extraído la resolución en cuestión. 
ii. Resumen de los hechos que la resolución haya considerado probados.

iii. Aspectos teóricos sobre los que se posicionase la resolución en cuestión y que guardasen relación directa con el concepto asignado al grupo.

9) A la vez que los alumnos hacían sus exposiciones en clase, el profesor responsable iba realizando las observaciones y comentarios pertinentes.

10) Todo el material de trabajo elaborado por los alumnos se encuentra disponible y puede ser consultado en la plataforma Moodle de la Universidad de Córdoba.

\section{RESULTADOS}

Los resultados conseguidos en este experiencia fueron los siguientes:

1) Se ha conseguido dar una dimensión práctica al aprendizaje que ha ayudado a consolidar los conocimientos del alumno, haciéndole ver la utilidad de los mismos en la vida real, y enfrentándoles a lo que va a constituir el modus operandi de muchos de ellos una vez finalicen sus estudios y se dediquen, como profesionales, al ejercicio del Derecho.

2) Ha sido, además, una forma de "obligar" al alumno al manejo de las bases de datos jurisprudenciales, permitiendo, al mismo tiempo, que se vayan familiarizando con el lenguaje del foro y con los documentos judiciales.

3) En la medida en que han tenido que extraer la información necesaria para elaborar los conceptos, les ha formado, igualmente, en la labor de discriminar aquellos aspectos que son relevantes, de aquellos otros que resultan accesorios, superando así la tendencia, tan propia de la técnica del "copia-pega", de hacer acopio de información innecesaria que nada tiene que ver con el problema cuya solución deben buscar.

4) Entendemos, asimismo, que ha contribuido positivamente a vencer el miedo a la exposición pública, al tiempo que ha servido para poner en valor, según han reconocido los propios alumnos, el esfuerzo del profesor en la transmisión de los conocimientos, pues han constatado lo necesario y, al mismo tiempo, lo difícil que resulta a veces realizar una exposición ordenada, clara y asequible, de conceptos complejos. Del mismo modo, han tomado conciencia de la dificultad que supone esa exposición cuando hay alumnos que no prestan atención o que perturban el silencio y el orden de la clase.

5) Es destacable, por último, que los resultados académicos en términos porcentuales de número de alumnos que han participado en la experiencia y han superado la asignatura han sido elevados, por lo que entendemos que la misma ha contribuido favorablemente a ello.

\section{CONCLUSIONES}

Con carácter general, la experiencia es altamente recomendable como metodología de trabajo, pues prepara al alumno para lo que va a ser su día a día una vez finalicen sus estudios de Derecho. Es especialmente aconsejable en aquellas disciplinas que forman parte de lo que se denomina "Derecho positivo" que, por estar sujetas a continuos cambios normativos, requieren del profesional del Derecho un proceso de constante actualización y puesta al día en sus conocimientos. En este sentido, puede afirmarse que los objetivos propuestos se han alcanzado, que los alumnos han quedado satisfechos y que la experiencia ha sido enriquecedora. 
No obstante, como en toda experiencia de esta naturaleza, junto a los aspectos positivos destacados como resultados, existen también elementos negativos que conviene resaltar.

Entre esos aspectos negativos, dejando al margen el incuestionable incremento de la carga de trabajo para el profesor, destacamos, sobre todo, el rechazo que este tipo de experiencias genera entre ciertos alumnos reacios a cualquier fórmula que no sea la explicación tradicional del temario por el profesor.

Conectado con lo anterior, el grado de compromiso de los alumnos no es siempre el deseable. Así, como en proyectos anteriores, ha habido alumnos que han preparado mejor las exposiciones de clase haciendo más fácilmente comprensibles los conceptos a sus compañeros, mientras otros se han limitado a reproducir lo que decían las sentencias sin entender en algunos casos, ellos mismos, qué era lo que estaban diciendo.

Como aspecto positivo, este curso, y a diferencia de lo sucedido en otros proyectos de innovación, el grado de cumplimento del cronograma previamente establecido ha sido del $100 \%$, por lo que no se han producido las distorsiones que otros años sí se habían observado.

\section{REFERENCIAS}

Barquín-Sánchez, J. (2016). Acercamiento práctico a la justicia penal como fundamento de la asimilación de conceptos jurídicos. En M. Turull-Rubinat \& E. Albertí-Rovira (Eds.), 74 experiencias docentes del Grado en Derecho (p. 42). Barcelona: Octaedro.

Ferrer-Martín de Vidales, C. (2016). Enseñanza a través de clases prácticas con manejo de bases de datos jurídicas". M. Turull-Rubinat \& E. Albertí-Rovira (Eds.), 74 experiencias docentes del Grado en Derecho (pp. 113-114). Barcelona: Octaedro.

López-Guede, J.M. (2015), Experiencia docente mediante la Metodología de Aprendizaje Basado en Problemas. Ikastorratza. e-Revista de Didáctica. Monográfico especial: Metodologías activas en la enseñanza universitaria, 14, 74.

Santaella-Quintero, H. (2016), La línea jurisprudencial como instrumento esencial para conocer el derecho. Docencia y Derecho. Revista para la docencia jurídica universitaria, 10, 2.

Vizcarro, C. \& Juárez, E. (2008), ¿Qué es y cómo funciona el aprendizaje basado en problemas? En García-Sevilla (Coord.), El aprendizaje basado en problemas en la enseñanza universitaria (pp. 18-19). Murcia: Servicio de Publicaciones de la Universidad de Murcia. 